\title{
Tobacco Harm Reduction
}

ISSN: 2689-2707

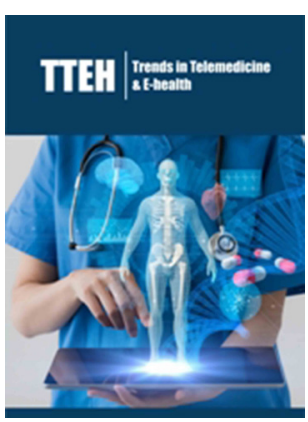

*Corresponding author: Somchai Bovornkitti, Department of Medicine, The Academy of Science, The Royal Society of Thailand, Bangkok, Thailand

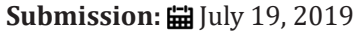

Published: 鲇July 26, 2019

Volume 1 - Issue 5

How to cite this article: Somchai B. Tobacco Harm Reduction. Trends Telemed E-Health. 1(5). TTEH. 000522. 2019. DOI: 10.31031/TTEH.2019.01.000522

Copyright@ Somchai Bovornkitti, This article is distributed under the terms of the Creative Commons Attribution 4.0 International License, which permits unrestricted use and redistribution provided that the original author and source are credited.

\author{
Somchai Bovornkitti* \\ Department of Medicine, Thailand
}

\begin{abstract}
Cigarette smoke contain approximately 250 different chemicals known to be harmful to human health. Thousands of harmful chemical substances produce by the combustion of tobacco. The health impacts such as cancer and chronic lung disease are not only associated with smokers but also people who are exposed to secondhand smoke. Tobacco Harm Reduction is a concept to minimize the impacts of tobacco on the individual and on society at large. A key component of this strategy is using alternative source of nicotine as a substitute to tobacco cigarettes. Electronic cigarette and heated tobacco are alternatives that might have potential in reduce harm from smokes. This paper elaborates on available research associated with electronic cigarette and heated tobacco with harm reduction and risk perspective.
\end{abstract}

Keywords: Tobacco cigarettes; Nicotine replacement therapy; E-cigarettes; Tobacco harm reduction

\section{Introduction}

Cigarette smoke contain approximately 7,000 different chemicals out of which 250 are known to be harmful to human health [1]. Tobacco smoking causes cancers, heart disease, stroke, diabetes, and chronic obstructive pulmonary disease (COPD) [2]. Smoking is responsible for 9 out of every 10 lung cancer deaths and 8 out of every 10 deaths from COPD. Smokers are more than 25 times more likely to develop lung cancer than non-smokers. Cigarette smoke contains high amounts of a number of different carcinogens which cause cancers anywhere in the body, with lung cancer and head and neck cancers being common among smokers. Cigarette smoke contains at least 69 known carcinogens, including nitrosamines, formaldehyde and several heavy metals. Smoking causes cancers of the lung, esophagus, larynx, mouth, throat, kidney, bladder, liver, pancreas, stomach, cervix, colon, and rectum, as well as acute myeloid leukemia [1]. In addition, smokers are more than twice as likely to have myocardial infarction as non-smokers. Smoking while pregnant can lead to low birth weight in newborns, cleft lips, stillbirths and sudden infant death syndrome (SIDS). Additionally, smoking affects the health of bones and the teeth and gums. For the aforementioned health related issues, the development of a tobacco harm reduction strategy is important to lower the overall usage of tobacco cigarettes [3-5].

\section{Efficacy of Anti-smoking Campaigns and Nicotine Replacement Therapy}

In the 1950s health science researchers established strong correlation between smoking and cancer. Subsequent public health outreach programs over the past seventy years included anti-smoking television, radio, and internet ad campaigns, raising taxes on tobacco products, and public smoking bans have all had impacts on the smoking rates, yet tobacco usage still remains a major public health concern [6].

Nicotine Replacement Therapy (NRT) provides a nicotine delivery system to tobacco users in a form that has reduced impacts to human health. They are principally promoted as a transition tool to for tobacco cigarette users to quit. NRT products include nasal sprays, patches, lozenges, inhalers and gum. Since their introduction to the market in the late 1970s, these forms of NRT's have been considered a useful tool for smoking cessation, however efficacy rates remain low. A review by Tang et.al. [7] indicated an approximation of $15 \%$ efficacy rate for the aforementioned NRT's. A primary reason for the limited efficacy, particularly among cigarette smokers, is that NRT's do not provide satisfaction of the smoking ritual and inhalation process. This concept was reinforced following the results of a recent 
study. The research indicated that among smokers, those who used e-cigarettes as a form of quitting tobacco had higher rates of abstinence from cigarettes than those who used other forms of NRTs or no cessation aid at all [8].

\section{Knowing Alternatives}

There are two forms, electronic nicotine delivery systems (E cigarette) and heated tobacco. The original e-cigarette is a battery-operated nicotine delivery system, commonly referred to as vaporizers or vapes. A vaporizer consists of four parts: a battery, a heating element to produce the vapor, a cartridge of the e-liquid solution which contains the nicotine and flavorings, and a mouthpiece to inhale the vapor. The liquid solution, and subsequently the vapor which is produced and inhaled, consists of glycerin and propylene glycol, along with nicotine and flavoring substances. Nicotine is addictive and can cause withdrawal symptoms but by itself is safer than the majority of chemicals in cigarette smoke. In addition to the inhaled vapor being safer than tobacco smoke, there is less interaction between secondhand smokers and the vapor, and thus less chance of the secondhand smoke to produce adverse health related consequences [9]. Several studies have shown that e-cigarettes are more effective in helping users quit smoking than any other type of NRT and more effective than nicotine-free placebo cigarettes [10].

The second form of alternatives are a "heated tobacco" or "heat-not-burn" tobacco product. Some studies also referred THS 2.2 which is one of heat-not-burn" tobacco product. THS 2.2 has extensive usage as a novel tobacco product in Japan and South Korea and has recently been approved by the United States Food and Drug Administration for consumer use. The product has three distinct components: a tobacco stick-made from tobacco powder, a holder into which the tobacco stick is inserted and heated by an electronically controlled heating blade, and a charger.

\section{Popularity of e-cigarettes}

Alternative forms of nicotine delivery systems have been available for decades. Nicotine patches, inhalation sprays, pills, chewing gum, and various other forms are available either over the counter or by prescription in many countries. While these products provide nicotine to the user, they do not provide the physical motions and social components of smoking, thus leading to a limited longterm success rate in preventing ex-smokers from relapse [11]. As a result, e-cigarettes have become extremely popular in the countries where they are legal as tobacco cigarette smokers look for a safer nicotine delivery system that has better long-term cessation rates. The THS 2.2 system maintains the flavor of tobacco while delivering a lower quantity of nicotine. This can allow the smoker a tool to slowly transition away from the addictive nature of cigarettes.

\section{Concerns of e-cigarette use}

E-cigarettes are somehow perceived as a gateway for tobacco use. While this has been proven true with other NRTs, it is unclear if e-cigarettes will have the same effect and further research is necessary. A recent study in the United Kingdom found that less than $1 \%$ of adults who were non-smokers had tried e-cigarettes.
This trend follows in the United States with a survey concluding that only $0.8 \%$ of non-smoking adults had tried e-cigarettes [11].

A major concern associated with e-cigarettes is youth usage. Nicotine is a highly addictive substance and can affect the development of children and teenagers. Despite the consensus that e-cigarettes are popular among young people, overall youth usage remains low in several study populations. As of August 2018, 2\% of 11-18-year old's in Great Britain used e-cigarettes weekly. In 2014, $70 \%$ of young people in Great Britain who had tried e-cigarettes had smoked tobacco cigarettes before, compared to $8 \%$ of young people who used e-cigarettes first followed by then tobacco cigarettes [12]. As the popularity of e-cigarettes has grown, the total number of smokers aged 18-24 has actually declined faster than other age groups [12].

Although e-cigarettes are safer than tobacco cigarettes, they are addictive. The use of e-cigarettes among people under the age of 18 should continue to be illegal and marketing strategies and access to e-cigarettes must be regulated and monitored. Marketing of e-cigarettes to minors must be prohibited to prevent an increase in youth usage. Youth usage must be monitored, and preventative strategies must be modified to address any changes in youth usage. Additionally, governments should consider limiting flavors of liquid e-cigarettes and nicotine levels [13-18].

\section{Conclusion}

Although the health risks associated with smoking combustible tobacco cigarettes is well established, many smokers refuse or have great difficulties quitting. E-cigarette and heated tobacco provide safer alternatives to tobacco cigarettes and can be used as part of a comprehensive approach towards tobacco harm reduction. E-cigarette and heated tobacco satisfy the smokers addictive cravings for nicotine as well as the physical motions of smoking. Multiple studies have been conducted to assess the risks associated with e-cigarettes and have concluded that they are a safer alternative to burnt tobacco cigarettes [12]. As a result, e-cigarettes should be legalized for adult smokers and comprehensive efforts should be made by regulatory bodies to ensure youth usage is minimized and research on the human health impacts must continue.

\section{References}

1. (2017) Harms of cigarette smoking and health benefits of quitting. National Cancer Institute, USA.

2. (2019) Fast facts. Smoking and tobacco use CDC, USA.

3. (2019) Smoking. British Heart Foundation, UK.

4. (2014) Smoking and respiratory diseases. CDC, Surgeon General Report on Smoking and Health $50^{\text {th }}$ Anniversary, USA.

5. (2018) Health effects of cigarette smoking. CDC, USA.

6. Warner KE (1977) The effects of the anti-smoking campaign on cigarette consumption. Am Jl Public Health 67(7): 645-650.

7. Tang JL, Law M, Wald N (1994) How effective is nicotine replacement therapy in helping people to stop smoking? BMJ 308(6920): 21-26.

8. Cummings KM, Hyland A (2005) Impact of nicotine replacement therapy on smoking behavior. Annu Rev Public Health 26: 583-599. 
9. Brown J, Beard E, Kotz D, Michie S, WestR (2014) Real-world effectiveness of e-cigarettes when used to aid smoking cessation: A cross-sectional population study. Addiction 109(9): 1531-1540.

10. Nitzkin JL (2014) The case in favor of e-cigarettes for tobacco harm reduction. Int J Environ Res Public Health 11(6): 6459-6471.

11. (2018) About electronic cigarettes (E-cigarettes). CDC, USA.

12. Fagerstrom KO, Bridgman K (2014) Tobacco harm reduction: The need for new products that can compete with cigarettes. Addictive Behaviors 39(3): 507-511.

13. (2019) Use of e-cigarettes among young people in Great Britain. ASH, UK.
14. Levy DT, Chaloupka F, Gitchell J (2004) The effects of tobacco control policies on smoking rates: A tobacco control scorecard. JPHMP 10(4): 338-353.

15. Mishra A, Chaturvedi P, Datta S, Sinukumar S, Joshi P, et al. (2015) Harmful effects of nicotine. Indian J Med Paediatr Oncology 36(1): 2431.

16. Doll R, Hill AB (2004) The mortality of doctors in relation to their smoking habits: A preliminary report. 1954. BMJ 328(7455): 15291533.

17. (2019) Tobacco. WHO Fact Sheets, WHO, Switzerland.

18. Glantz SA, Bareham DW (2018) E-cigarettes: Use, effects on smoking, risks, and policy implications. Annu Rev Public Health 39: 215-235. 